http://dx.doi.org/10.18359/ravi.2682

\title{
Nuevas funciones docentes para la gestión del conocimiento en la web social $^{1}$
}

Leonardo Emiro Contreras Bravo² \& Karolina González Guerrero ${ }^{3}$

Universidad Francisco José de Caldas - Universidad Militar Nueva Granada, Colombia

Recibido, diciembre 18 de 2016

Concepto evaluación, enero 17 de 2017

Aceptado, enero 30 de 2017

\author{
Referencia: Contreras Bravo, L. \& González Guerrero, \\ K. (2017). "Nuevas funciones docentes para la gestión \\ del conocimiento en la web social". Revista Academia y \\ Virtualidad, 10, (1), 95-106
}

\section{Resumen}

El docente en la actual sociedad de la información ha tenido que cambiar su rol debido a la incidencia de las tecnologías de la información y la comunicación en el contexto de la educación superior. En ese sentido, su quehacer pedagógico y didáctico se ha ido transformando en relación con dinámicas mucho más colaborativas con sus educandos. Así, para indagar estas prácticas colaborativas enmarcadas en el concepto de docente-prosumidor, se hace uso de una metodología de corte cualitativo - basado en la teoría fundamentada en el marco metódico axial y selectivo-orientada hacia una reflexión de las implicaciones comunicativas y colaborativas del docente en la web 2.0 dentro del escenario educativo. De igual modo, el resultado del manuscrito se traduce en la caracterización y ejemplificación de este rol del docente-prosumidor, al analizar a modo de discusión la necesidad de un proceso formativo con el fin de desarrollar pedagógica y críticamente este papel en la educación superior.

Palabras clave: características del prosumidor, docente-prosumidor, educación superior, empoderamiento, web 2.0.

\footnotetext{
${ }^{1}$ Resultado de proyecto ING 2109, financiado por Vicerrectoría de Investigaciones, Universidad Militar Nueva Granada (2016).

${ }^{2}$ Docente Investigador, Universidad Francisco José de Caldas; Ingeniero Industrial, Magíster Procesos. 1.contreras@udistrital.edu.co

${ }^{3}$ Docente Investigadora, Universidad Militar Nueva Granada; Licenciada en Electrónica; Magíster en

Educación; Doctora en Educación. karolina.gonzalez@unimilitar.edu.co
} 


\title{
New teaching roles regarding knowledge management in the social web
}

\begin{abstract}
The teachers in the current information society have had to change their role due to the impact by the ICTs upon the higher education. In that sense, their pedagogical and didactic task has been changing in relation to more collaborative dynamics with their trainees. Thus, to investigate these collaborative practices within the concept of teacher-prosumer, we have used a qualitative methodology based upon a methodical axial and selective framework towards a reflection upon collaborative and communicative effects by the teacher in web 2.0 within the education environment. Also, the result of this paper is translated into a characterization and an exemplification of the role by a teacher-prosumer, by discussing the need for a learning process in order to develop pedagogically and critically this role in higher education.
\end{abstract}

Keywords: prosumer characteristics, teacher-prosumer, higher education, empowerment, web 2.0 .

Novas funções docentes para a gestão do conhecimento na web social

\section{Resumo}

$\mathrm{Na}$ atual sociedade da informação o docente teve de mudar seu papel devido a incidência das tecnologias da informação e a comunicação no contexto da educação superior. Neste sentido, seu fazer pedagógico e didático tem vindo se transformando com relação as dinâmicas muito mais colaborativas com seus estudantes. Assim, para indagar nessas práticas colaborativas enquadradas no concepto de docente-prosumidor, se utiliza uma metodologia de tipo qualitativo - baseado na teoria fundamentada no quadro metódico axial e seletivoorientada na direção de uma reflexão das implicações comunicativas e colaborativas do docente na web 2.0 dentro do cenário educativo. Do mesmo jeito, o resultado do manuscrito traduz-se na caraterização e exemplificação deste papel do docente-prosumidor, ao analisar como discussão a necessidade de um processo formativo a fim de desenvolver pedagógica e criticamente este papel na educação superior.

Palavras chave: caraterísticas do prosumidor, docente-prosumidor, educação superior, empoderamento, web 2.0. 


\section{Introducción}

De acuerdo con investigaciones realizadas por el grupo Pedagogía y Didáctica en la Educación Superior [PYDES] acerca de la influencia de las tecnologías de la información y la comunicación [TIC] en la educación superior, el rol del docente, bien sea en el uso de ambientes de aprendizaje virtuales o aspectos de la web 2.0, reconfigura toda una acción pedagógica y didáctica en torno a facilitar el aprendizaje del educando (González, Padilla \& Rincón, 2012). Por ello, y a la luz de lo expuesto por Giurgiu \& Bàrsan (2008), las funciones del docente se perfilan en aspectos metacognitivos, creativos $\mathrm{y}$ autónomos que permitan desarrollar un aprendizaje significante en el educando. Así las cosas, subyace la necesidad de caracterizar el docente-prosumidor (acrónimo de las palabras productor y consumidor), como una figura emergente en el contexto de la educación superior, contribuyendo a procesos más dialógicos e interactivos, supeditado a una transición de paradigmas de enseñanza a otros de aprendizaje.

\section{Método}

Teniendo en cuenta el objeto del estudio, el cual es el docente-prosumidor, la investigación adopta un enfoque cualitativo (Sautu et al., 2005; Miguel Valles, 2003) para establecer relaciones o tendencias del concepto de la web 2.0 y el surgimiento de la figura prosumidora a través de la exégesis y categorización de textos con base en la teoría fundamentada. En este tipo de metodología, se consideran varios documentos primarios y se analizan bajo un ejercicio interpretativo. De tal manera que los datos clasificados, codificados y representados en redes semántica-categorías y subcategorías de análisis-, sean contrastadas con el método de la comparación constante (Valles, 2002) y apoyo técnico del software Atlas ti.

En referencia a lo expuesto anteriormente, la búsqueda, la clasificación y la codificación de los documentos se realizó con una definición operativa de la categoría de análisis: "Características del docente-prosumidor", en tanto permitiera develar la densidad y fundamentación de subcategorías, tales como: (1) web 2.0 en educación superior, (2) rol del docente en educación superior, y (3) ejemplos de acciones prosumidoras (en Atlas ti. se traduce en la cantidad de citas compartidas por varios códigos). De ahí que las concurrencias entre códigos a la luz de la Teoría fundamentada (Grounded Theory; Strauss \& Corbin, 2002, p.13) con apoyo del software Atlas ti, se reflejen en tendencias útiles para establecer marcos de referencia e interpretación. En suma, Para Strauss \& Corbin (2002), la teoría fundamentada se puede utilizar en un mayor entendimiento de cierto fenómeno emergente y así poder profundizar en él en términos descriptivos, explicativos o en este caso interpretativos.

\section{Búsqueda y selección de la información}

La búsqueda de los documentos se realizó a través de bases de datos virtuales especializadas como: ScienceDirect, Scielo, Redaly, Dialnet, Google académico, entre otras. De lo anterior, se contemplaron 105 documentos (relacionados con la web 2.0 en educación superior) de los cuales 25 se referían directamente al objeto de estudio: docentes-prosumidores.

\section{Proceso de codificación}

De manera previa al proceso de codificación o clasificación de la información, se formularon las categorías: (1) web 2.0 y la educación superior y (2) caracterización del docente-prosumidor, de las cuales se fueron alimentando los códigos de análisis (véase figura 2) como referente para la clasificación de la información por interpretar desde las categorías de análisis y/o emergentes. Estas categorías se construyeron en principio, desde la discusión y conceptualización de teorías consolidadas del tema web 2.0 y prosumidores (Alvin Toffler y Marshall McLuhan), además 
de investigaciones efectuadas sobre el tema por el grupo de investigadores.

En la primera fase de codificación (abierta), se aplicó una clasificación inicial de los datos que permitiera reducir los documentos a unidades analizables, es decir, a fragmentos de texto con significado (citas), cuyo fin es organizar y dotar de sentido las lecturas hechas sobre los documentos primarios. Posteriormente, en la interpretación de los datos por cada categoría, fue necesaria la codificación axial para relacionar códigos con citas compartidas. Finalmente, como segunda fase, se adicionó el método de codificación selectiva, puesto que con mayor entendimiento del objeto de estudio se realizaron comparaciones de los códigos y sus respectivas citas, leyendo a profundidad y de ser necesario, ajustando las subcategorías en nuevos códigos y jerarquías.

\section{Resultados}

El aporte del estudio, en términos de resultados, se presenta como una reflexión y discusión en torno a la caracterización y ejemplificación de la acción prosumidora de los docentes en el marco de la educación superior. Así pues, el enfoque de los resultados está orientado a una perspectiva crítica y propositiva sobre la importancia de un componente pedagógico y didáctico para abordar el papel del docente en medio de la incidencia de las tecnologías de la información y la comunicación [TIC] y la web 2.0 en la educación superior. Para ello, se desarrolla un acercamiento a la caracterización del docente-prosumidor, las dificultades que se pueden presentar y un panorama contextual de ejemplos, que han logrado permear el escenario educativo por medio de la web 2.0 a través de la labor de prosumidores en proyectos colaborativos, autónomos y trascendentes para facilitar el aprendizaje.

\section{Nuevas funciones asociadas al docente en la web social}

Las funciones del docente harán mención en la propuesta de formación, a las acciones que debería desarrollar el profesor-prosumidor, cuyo quehacer pedagógico desde la concepción socio crítica enfrenta retos que no sólo están en el linde del marco disciplinar, sino además se sustentan en el acompañamiento y orientación del estudiante en aras de establecer una conciencia humanística y centrada en aspectos investigativos para la calidad educativa. Por lo tanto, la labor del docente se diversifica y sus actividades convergen en relaciones e integraciones que incluyen apoyos y medios sustentados en TIC.

Este proceso de formación del docente para afrontar la educación b-learning bien tiene también un impacto, en las consideraciones institucionales, donde una prioridad sea motivar al docente en su capacitación (Marqués, 2000). Puesto que si se espera que el docente afronte un cambio dictaminado no sólo por el marco social- educativo, sino por las demandas que impone las TIC, es relevante afrontar el hecho de que estas nuevas funciones y tareas que se le delegan al maestro de espacios mixtos sean encaminadas no únicamente a facilitar recursos al estudiante, también para comprender la importancia de romper paradigmas con respecto a la figura de transmitir unilateralmente saberes por parte del educador, en la cual se espera es un acompañamiento a la formación integral del estudiante (González, Rincón y Contreras, 2013b).

Teniendo en cuenta el análisis hecho en el marco referencial respecto a la descripción del docente en el contexto b-learning, en el análisis de los datos obtenidos por encuestas y entrevistas frente a la clasificación de las funciones y el análisis a profundidad del surgimiento del docente-prosumidor, a manera propositiva se plantean una serie de funciones que el docente debería articular junto con su desempeño en el aula, con la finalidad de establecer un epicentro de transformación crítica basado en la investigación e interacción efectiva. 


\section{Función de comunicación}

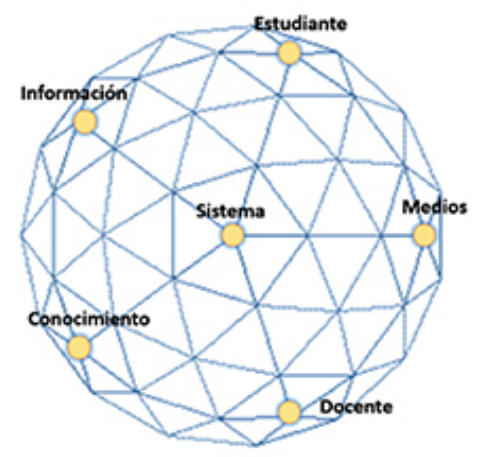

Figura 1. Esquema de conexión de la función de comunicación dialógica del docente-prosumidor.

El docente-prosumidor tiene por condición una comunicación dialógica, abordada desde su perfil de productor-expresión y el usuario-análisis, por ende la comunicación no es unidireccional; más aún, no convoca únicamente lo lingüístico, sino la capacidad de comunicación visual y simbólica. La comunicación de la acción prosumidora va más allá de diálogos y conversaciones con otros factos, trasciende a una relación intersubjetiva, producto del impacto de las TIC y los desarrollos informáticos del momento, además de múltiples teorías comunicativas que se van anclando y objetivando en el devenir social. La comunicación en este ámbito debe ser totalmente conexa, multidireccional entre todos los factores que intervienen en este proceso (el docente, el estudiante, los medios TIC, el sistema, el mensaje, el conocimiento y la información).

Area y Pessoa (2012), al respecto, recalcan la necesidad de una alfabetización de variados códigos y elementos comunicativos transformados por el impacto tecnológico, traduciendo esta práctica en una competencia mediática para la búsqueda, selección e interpretación de información, para luego ser dialogada, refutada y asociada al sistema comunicativo en la interacción y formas de validar dicha información en conocimiento. Por tanto, el sistema comunicativo ya no puede basarse en el esquema de emisor, mensaje y receptor (Aparici, 2011), pues además de estos factores y sumados a ellos el componente de retroalimentación como factor de regulación, permutan para que lo informativo se incline a lo formativo a través de pautas para una dinámica participativa, concibiendo un modelo dialógico y conversacional que toma auge en el campo educativo a través del concepto prosumidor para denotar la condición de un educando con posibilidades de autorregular, gestionar y alternar mensajes e información por medio de la dualidad de emitir y recibir sin dejar de lado la contextualización de la información.

Este factor comunicativo tiene incidencia en los aspectos de diseño, distribución y empleo de diversos modos de dar a conocer la información, puesto que emplea un escenario distinto y complementario para reforzar dese el punto de vista educativo una facilitación del aprendizaje.

\section{Función de interacción}

Ante el incremento de prácticas prosumidoras, se devela la importancia de la interactividad, consolidada a mediados de la década de 1980, producto de los esfuerzos mancomunados de los usuarios y productores, las tecnologías y sus recursos han posibilitado la mediación, comunicación y colaboración entre cadenas y estructuras de grupos sociales para una 
finalidad común.

En palabras de Araya (2008), la interactividad en los prosumidores se puede catalogar de la siguiente manera: intervención por parte del usuario sobre el contenido, transformación del espectador en actor, diálogo individualizado con los servicios conectados, acciones recíprocas en modo dialógico con los usuarios o en tiempo real con los aparatos (cada uno de los comunicadores responde al otro o a los otros). La interactividad debe ser algo inherente, no sólo a las implicaciones tecnológicas, sino además a la forma de comunicarse socialmente, en especial entre los prosumidores y usuarios.

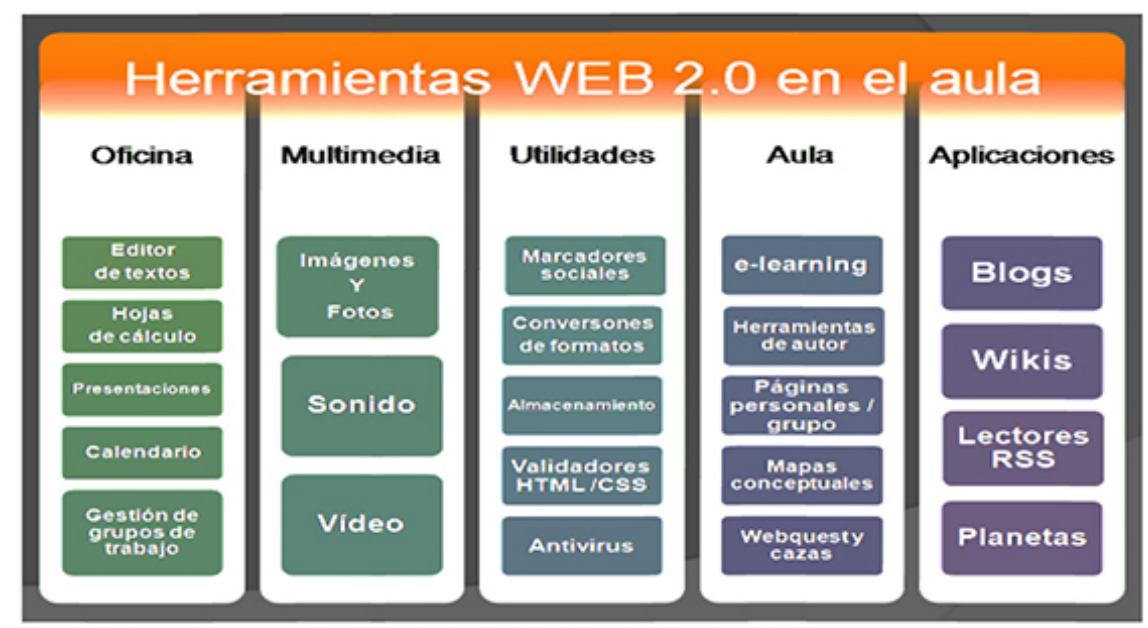

Figura 2. Herramientas 2.0 que facilitan la interacción entre docente y estudiantes en la web social. Fuente: Araya (2008, p.55)

La intervención por parte del usuario sobre el contenido puede darse a través de la participación e intervención como algo más allá de la opinión, en cuanto se modifican prácticas y contenidos a través de la inclusión de experiencias y temas propios. Esta intervención del prosumidor trae consigo la multidireccionalidad o principio de hibridación, el cual converge en las acciones de emisión y recepción en una co-construcción de mensajes concomitantes a un sentido y significado compartido. Por último, se puede caracterizar el principio de intercambio y potenciación como la facultad de consolidar redes y entramados de comunicación que van más allá del agrupamiento, permitiendo la conmutación de significados al desarrollar nuevos contenidos, producto del intercambio, la asociación y la libre expresión.

La interactividad a su vez es posible siempre y cuando al prosumidor se le den las herramientas, la libertad y la oportunidad de crear y usar los recursos para alcanzar sus metas. En ese sentido, la elección es un factor sustancial en las formas de innovar del prosumidor, el no circunscribirlo en una sola ruta le lleva a explorar por diferentes caminos, comprendiendo cuál de ellos es el más adecuado para resolver problemáticas.

El perfil del prosumidor posee la capacidad de entrar y salir del papel de consumidor, esto siempre y cuando sea desde una postura reflexiva, pues la difusión de contenidos en internet: documentos, imágenes, videos o podcast no lo hacen un prosumidor, es más bien una contribución significativa para otros pares o personas que están actuando como usuarios. Giurgiu y Bàrsan (2008), al respecto, agregan la importancia de la creación de contenidos para la transformación social; el público, consumidor o masas no pueden ser pasivas ante la recepción, deberán participar como ciudadanos, más allá de usuarios que digieren toda la información producida en diversos medios de comunicación. El blog, la wiki y las redes sociales en principio 
constituyen opciones para plasmar la posición frente a algo. No obstante, las plataformas de código abierto, simulaciones, y comunidades prosumidoras de maneras más avanzadas pueden aplacar jerarquías y oligopolios instaurados por la misma paradoja tecnológica de la manipulación y control de los medios masivos de información y comunicación.

De lo anteriormente dicho: la interactividad, la innovación y el proceso crítico de creación de contenidos, el docente al asumir el rol de prosumidor, habrá de entender esta dimensión reflexiva, en principio, a través de un rol del usuario, estableciendo un paralelo a posteriori de conocer las necesidades como estudiante y las contribuciones esenciales en su labor de educador. Como usuario de las TIC, el docente encuentra una iniciación concordante con la solidaridad de incluir las experiencias y dificultades de sus alumnos; wikis, foros y redes sociales permiten formular recursos potencialmente didácticos, orientados con unas finalidades y objetivos de aprendizaje particulares al ambiente educativo.

\section{Función de empoderamiento}

Otra función característica del prosumidor convoca una acción de empoderamiento, cuyo valor intrínseco radica en la defensa de los derechos (Sánchez y Contreras, 2012), de los valores y del respeto a la acción ciudadana de transformar su entorno y mejorar la calidad de vida. El ciudadano común, el experto, el profesional e inclusive las personas con limitaciones, son capaces de transformar y aportar al desarrollo sociocultural; por ende, el cambio de rol de simple receptor debe ser gradual conforme su participación creativa, sea medular en la acepción de libertad de expresión e intervención ante aspectos que entran en detrimento en la dinámicas ética, políticas y estética.

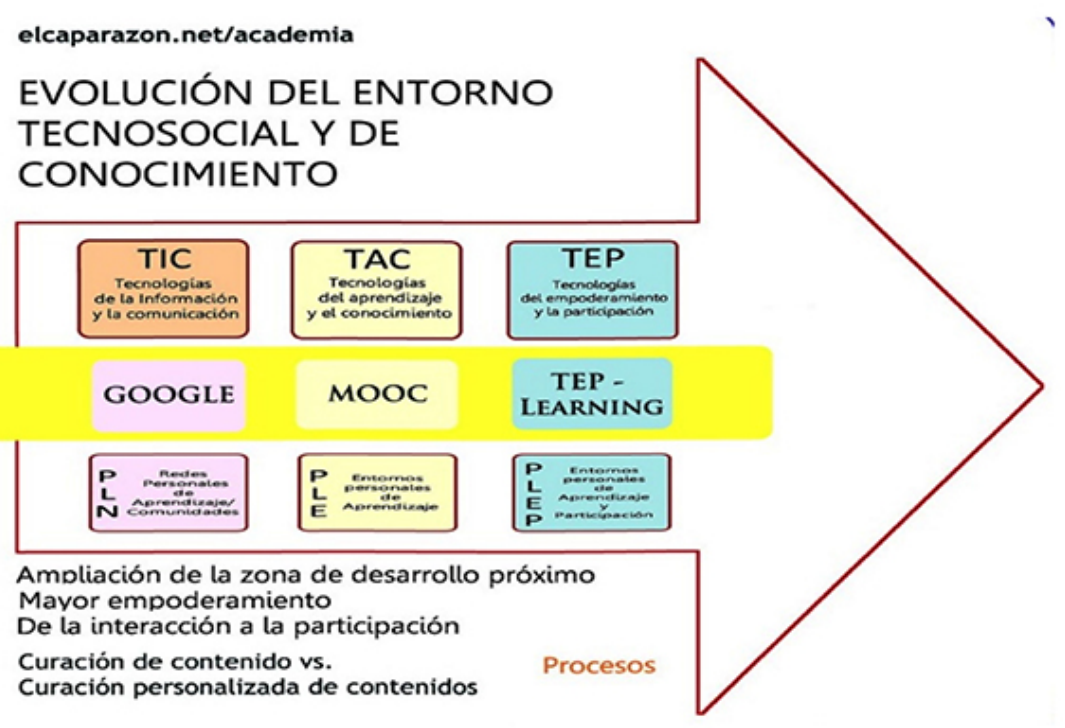

Figura 3. Evolución de las TIC a las TAC y a las TEP. Fuente: El caparazón blog 
La búsqueda de un proyecto personal radica en la independencia y la opción de conocer libremente desde la autonomía a la hora de buscar lo que se considere de interés o prioritario para el aprendizaje continuo. Islas (2008) añade a esta perspectiva defensora la relevancia de la emancipación como condición valiosa del progreso personal, indistintamente de la educación formal, la garantía de una interiorización de dicha información que conlleva a una elaboración del perfil prosumidor.

El mando atribuido al consumidor no puede ser solamente la deliberación del marketing, pues la voz pasiva de esta figura no debe servir para callar otras voces, sino que debe estimular el aliento de otras masas que tengan por referente la toma de decisiones de manera consciente y responsable, sabiendo que la resultante tiene un impacto negativo o positivos sobre otros. Por eso se habla de multidireccionalidad comunicativa (Alonso, 2011), pues en medio de esta emancipación coexiste el diálogo entre el usuario y la jerarquía y la posibilidad creciente de un diálogo inter-pares.

Los prosumidores se empoderan toda vez crean, construyen e innovan, dejando de lado ese rol primigenio de esperar a que llegue a él lo producido por otros, la inconexa forma de hacer contenidos y servicios para otros era cuestión de eruditos; sin embargo, ahora en el polimorfismo todos tiene la condición de incentivar o crear algo, pero no algo aislado o resultante de un esfuerzo individual, pues el prosumidor es una razón de la comunidad prosumidora. No se puede refutar algo que no se conoce, es el viejo adagio que consecuentemente abre el camino al empoderamiento del prosumidor, pues más allá de producir, provee iniciativas para que otros hagan algo para el cambio, una transición eximida de la neutralidad, ya que siempre hay una postura política, acorde a desvirtuar o legitimar poderes, inclusive los tecnológicos, pero "[...] surge el prosumer, que conoce cómo se realizan los medios y las mediaciones, que exige el lugar que se ha ganado para ejercer una ciudadanía vigilante ante las injusticias" (Sánchez y Contreras,
2012: 80), acto seguido a la reivindicación de una acción prosumidora empoderada hacia la necesidad de alfabetizarse y crear un pensamiento crítico hacia la transformación social.

\section{Función educadora}

En este estudio, la llave maestra del cambio a un modelo pedagógico autónomo y facilitado en perspectivas pedagógicas diversas, la tiene el docente, pues en el profesorado están las pautas a una transición reflexiva de la importancia de vincular al educando en el diseño de los procesos educativos. Es el docente quien da la posibilidad de realizar cambios, ofrecer distintas posturas, significaciones y creaciones mancomunadas, sin perder la ruta de aprendizaje trazada desde los fines curriculares e institucionales designados por la universidad o el programa de formación. Así pues "[...] una pedagogía basada en esa disposición a la coautoría, a la interactividad, requiere la muerte de un modelo de profesor y el nacimiento de un docente democrático y plural" (Aparici y Silva, 2012: 57).

Para un aprendizaje de este estilo, es fundamental un cambio de posicionamiento del docente, pues pasa de ser el eje central a considerar que todo el grupo está en constante aprendizaje, cuyo referente es motivar y aproximar al educando a un proceso creativo, flexible y reflexivo a través de la interacción, conmensurado por las oportunidades comunicativas que brindan las TIC. Esta visión del docente, se traduce en estudiantes prosumidores, más que receptores y consumidores de información, se vuelven personas productoras de significados por medio de la dinámica de vincular intereses, problemas e inquietudes representativas de la realidad construida por las mediaciones culturales, cognitivas y actitudinales vividas en la cotidianidad.

Este paradigma de formación, tendiente al aprendizaje y no a la enseñanza, se ha ido afianzando por medio de las implicaciones de las TIC, más exactamente por el auge de la web social, lo cual lleva a analizar las formas 
y prácticas que han cambiado a posteriori de incluir estos recursos en el escenario de la educación superior. De ahí que se distinguen las siguientes permutaciones del alumno y docente en situación de rol prosumidor:

- Modificaciones en los recursos y materiales de aprendizaje: los accesos a múltiples materiales $\mathrm{y}$ en distintos formatos abren la posibilidad de abarcar distintos ritmos de aprendizaje. La búsqueda en bibliotecas, es importante, pero además lo constituye la consulta de bases de datos, plataformas educativas, redes sociales, repositorios y sistemas de comunicación variados en la diversidad multimedia.

- Inclusión activa del estudiante: no basta con acceder a la información, es básico que docentes $\mathrm{y}$ estudiantes puedan modificarla, gestionarla y estructurarla de una manera sistemática para la aprehensión. Ambos están en la labor de aportar, compartir y socializar de manera multidireccional discusiones sobre el análisis de lo indagado.

- Experiencias individualizadas: tanto estudiante como docente podrán incluir experiencias de aprendizaje, se deberán respetar las singularidades y se podrá establecer diferentes actividades centradas en las habilidades y desventajas de cada persona.

- Experiencias colaborativas: no es necesario fijar lo colaborativo en el trabajo en equipo; por ende, no basta sólo con grupos focalizados dentro del colectivo en general. Es menester de este apartado señalar que lo colaborativo desde la corriente social-constructivista busca que los integrantes del grupo tengan presente que el aprendizaje es dependiente del otro y viceversa, por lo cual hay una serie de responsabilidades o roles para alcanzar los objetivos del grupo. Lo colaborativo debe extrapolarse fuera de las aulas, en comunidades abiertas, sin etiquetas y si es posible con diversas personas externas al ambiente institucional.
- La resolución de problemas: en este punto es clave enunciar que no se solucionan problemas, el resolverlos no es producto de pruebas estandarizadas y posposiciones lógico-matemáticas, pues más allá está una estrategia didáctica confinada a develar proceso creativos, proyectuales y conceptuales para el planteamiento y resolución de dichos problemas.

Al aprender en simultánea educadores y educandos, el meta-aprendizaje se convierte en una forma de aproximarse al conocimiento, aspecto fundamental en la educación superior, la cual empieza por diseñar currículos que den cabida a la figura del prosumidor a través del análisis de pares y entre pares de situaciones consensuadas para modular procesos formativos adheridos a las experiencias $\mathrm{y}$ resultados de los estudiantes. Es inevitable: la economía invisible o del conocimiento arroja a la educación superior unas obligaciones de formación más reflexivas y críticas, modos de aprender diversificados para una toma de postura argumentada y centrada en las bases del cambio, aquel que en el tan anhelado constructivismo no pudo alcanzar y donde las competencias empiezan a incursionar sin mayor vestigio positivo.

\section{Función de la evaluación}

Esta función contempla por parte del docente, el diseño, la estructuración y el seguimiento de la evaluación que se efectúa en el curso. El docente para ello contará con estándares de calidad y normatividad asociada al uso de las TIC, teniendo en cuenta procesos de evaluaciones de orden formativo y democrático para que haya consenso y retroalimentación de los procesos que se llevan a cabo por parte del grupo de estudiantes en su formación bajo el contexto b-learning.

Así pues, el docente en el marco de su función evaluadora debe ser consciente de que la evaluación no sólo recae en el aprendizaje del estudiante, sino que deberá contemplar elementos como los contenidos del curso, las 
actividades que se desarrollan, la calidad de la interacción entre otros aspectos que pueden llevar a determinar una formación de calidad, seguida desde los desempeños de los diversos roles del docente y del estudiante.

La función evaluativa que proporciona el docente debe estar en constante marcha a lo largo de todo el curso como un recurso dinámico (Sierra, 2009) con el fin de determinar si los objetivos de la formación se están cumpliendo, sin esperar hasta la culminación del proceso, instancia en la cual ya sería obsoleto el análisis y reflexión con base en las dificultades y fortalezas inherentes al proceso pedagógico. Por ende, a continuación se caracterizan las acciones y actividades que el docente debe tener en cuenta para consolidar esta función:

- Acción de diseño y planteamiento de la evaluación: el diseño conlleva a que el docente obtenga información, de tal manera que le permita dar una exhortación sobre los prospectos evaluativos que se requieren incluir dentro del curso; por consiguiente, con base en ese análisis, el docente arma una carta de navegación en evaluación, que consistirá en un proceso constante y detallado sobre los pormenores de interacción, adaptación y seguimiento que se presentan en el curso, con expectativas a la adquisición de una serie de competencias por parte del alumno a través de estrategias que puedan dar una visión del estado del curso y los aspectos por mejorar (Martínez et al., 2006).

- Acción de evaluación del aprendizaje: al hacer clarividencia de una evaluación que no es propia únicamente del acto de calificar, sino que enmarca un proceso enriquecido para la formación no sólo del estudiante, también se hace pertinente para la reflexión y el mejoramiento del curso de formación en general (Martínez et al., 2006). En consecuencia, su diseño debe pretender estimular y colaborar con el estudiante para construir conocimiento. En ese orden de ideas, el docente ofrecerá diversos tipos de evaluación supeditados por estándares en TIC y diseño curricular para llegar a orientar al estudiante en el proceso pedagógico.

- Acción de autoevaluación y coevaluacion: ésta corresponde a la evaluación de la praxis ejercida por el mismo docente y bajo la figura del prosumidor, cuya evaluación está ligada a la coevaluacion por parte del estudiante y los pares. Esta acción pretende la reflexión cíclica de los procesos educativos desarrollados; asimismo, pretende ser continua y formativa, de hecho no existe una sin la otra.

\section{Conclusiones}

Los códigos elaborados de los que habla Basil Bernstein, como un sistema de significación universal (1990), se pueden extrapolar a la condición académica manejada en las instituciones de educación superior (IES), las cuales de cierto modo confinanaquellos códigos marginales o restringidos que se traducen en microesferas contextualizadas, experiencias cotidianas y pensamientos del común que pueden ayudar en el marco representacional a adquirir un conocimiento significativo. En esa medida, la web 2.0 se convierte en un puente cognitivo y actitudinal que supera la forma pedagógica selectiva, enunciada por Bernstein, a través de los componentes de colaboración, interacción y autonomía (1990), para lograr desde un ambiente mediático la posibilidad transaccional cultural y un conocimiento significativo a través de experiencias dialógicas educativas. De este modo, es menester de este manuscrito resaltar la importancia de la web 2.0 más allá de sus componentes tecnológicos y técnicos, en tanto se propone un escenario de discusión y reflexión en torno a cómo se articula pedagógicamente la web 2.0 y desde una postura crítica cómo puede incidir en la calidad e igualdad educativa.

Al realizar un análisis e interpretación de los datos acorde al objeto de estudio, en este caso, el docente con atributos de prosumidor en el contexto de la educación superior, se 
encuentra que los documentos primarios y las bibliografías acordes al tema son escasos, pues si bien la figura de prosumidor se destaca en otros ámbitos como el marketing, la publicidad, la gestión de contenidos en la red y el desarrollo de elementos multimedia, en el campo educativo no se ha profundizado en la figura del docente-prosumidor, la cual se muestra preponderante en el marco de la inclusión de las tecnologías de la información y la comunicación [TIC], y específicamente en la revolución de la web 2.0 en escenarios presenciales y virtuales para generar conocimiento significativo en la educación superior.

A raíz de esta problemática del docenteprosumidor, desde los referentes de Alvin Toffler (1980) y Marshall McLuhan (2009), y acorde con la interpretación a través de la revisión de categorías como la caracterización y ejemplificación del docente-prosumidor, se puede constatar que es necesario un proceso de formación continua de los docentes para alcanzar esta condición prosumidora, pues aunque se reconocen avances en lo concerniente a la actualización y capacitación en el uso de herramientas, recursos y plataformas en la parte práctica y operativa para producir e integrar tecnologías en su quehacer profesional, también es evidente el desconocimiento pedagógico y teórico que implica actitudes, componentes éticos y estéticos del prosumidor para dotarlo de unas didácticas específicas y acordes a los objetivos de aprendizaje que se desean alcanzar.

\section{Referencias}

Alonso, M. (2011). El plan de Marketing digital (El consumer se ha transformado en prosumer). Madrid: IE Business School, pp. 234.

Aparici, R. (2011). Principios pedagógicos y comunicacionales de la educación 2.0. Revista digital la educ@ción, 145, 1-14. Recuperado de

http://www.educoas.org/portal/La_
Educacion_Digital/laeducacion_145/articles/ Roberto_Aparici.pdf

Araya, D. (2008). The democratic turn: Prosumer innovation and learning in the knowledge economy. En M.A. Peters \& R. Britez (Eds.) Open education and education for openness (pp. 17-32). Rotterdam: Sense.

Area, M. y Pessoa, T. (2012). De lo sólido a lo líquido: las nuevas alfabetizaciones ante los cambios culturales de la Web 2.0. Revista digital la educ@ción, 145, 13-20. Recuperado de http://www.revistacomunicar.com/pdf/ preprint/38/01-PRE-12378.pdf

Bernstein, B. (1990). Class, codes and control: the structuring of pedagogic discourse (vol. 4). Madrid: Morata, pp. 172.

Giurgiu, L. \& Bàrsan, G. (2008). The prosumer - core and consequence of the web 2.0 era. Revista de informática Sociala, 5 (9), 53-59. Recuperado de http://www.ris.uvt.ro/wpcontent/uploads/2009/01/giurgiubirsan.pdf

González, K., Padilla, J.E. \& Rincón, D. (2012). El docente en contextos b-learning. Bogotá, D.C.: Universidad Militar Nueva Granada, pp. 45.

Islas, J.O. (2008). El prosumidor. El acto comunicativo de la sociedad de la ubicuidad. Palabra clave, 11(1), 29-39. Recuperado de http://octavioislas.files.wordpress. com/2009/09/octavioislas_2008_coneicc.pdf

McLuhan, M. (2009). Comprender los medios de comunicación: las extensiones de ser humano. Buenos Aires: Paidós, pp. 211.

Salinas, J. (1997). La educación en la sociedad de la información. Barcelona: Santillana, pp. 145 .

Sánchez, J. \& Contreras, P. (2012). De cara al prosumidor, producción y consumo empoderado a la ciudadanía 3.0. ICONO, 
10 (3), 62-84. Recuperado de http://www. icono14.net/ojs/index.php/icono14/article/ view/210

Sautu, R., Boniolo, P., Dalle, P. \& Elbert, R. (2005). Manual de metodología. Buenos Aires: Colección Campus Virtual, pp.104.

Strauss, A. \& Corbin, J. (2002). Bases de la investigación cualitativa. Técnicas y procedimientos para desarrollar la teoría fundamentada. Medellín: Editorial Universidad de Antioquia, pp. 213.

Toffler, A. (1980). La tercera ola. Bogotá: Plaza \& Janes, pp. 86.

Valles, M. (2003). Técnicas cualitativas de investigación social reflexión metodológica y práctica profesional. Madrid: Editorial síntesis, pp. 122.

(2002). Ventajas y desafíos del uso de programas informáticos (e.g. ATLAS. ti y MAXqda) en el análisis cualitativo. Una reflexión metodológica desde la grounded theory y el contexto de la investigación social española. Recuperado de http://public. centrodeestudiosandaluces.es/pdfs/S200105. pdf 